\title{
REAL-TIME SYSTEM BASED ON FEATURE EXTRACTION FOR VEHICLE DETECTION AND CLASSIFICATION
}

\author{
Zakaria Moutakki $^{1,2}$, Imad Mohamed Ouloul', Karim Afdel ${ }^{1}$, Abdellah Amghar ${ }^{1}$ \\ ${ }^{1}$ Laboratory of Metrology and Information Processing, Department of Physics, Faculty of sciences, \\ University Ibn Zohr, B.P 8106, Agadir, Morocco \\ ${ }^{2}$ zakaria.moutakki@edu.uiz.ac.ma
}

\begin{abstract}
Today, Road traffic video surveillance becomes the centre of several concerns. It presents an important way for analysis of road traffic in highways. Road traffic video surveillance can help to resolve many problems which can influence road safety. This paper presents a real-time management and control system which serve to analyze road traffic using a stationary camera. The proposed system can measure the quantity and characteristics of traffic in real time based on three modules, segmentation, classification and vehicle counting. Our contribution consists of developing a feature-based counting system for vehicle detection and recognition under the conditions which present a challenge in recent systems, such as occlusions, and illumination conditions. Our method can perform vehicle detection and classification by eliminating the influence of many factors on system efficiency. The obtained results show that the system proposed in this paper provides a counting rate higher than that of some existing methods.

Keywords: Intelligent transportation systems, Vehicle detection, Occlusion handling, Vehicle classification, Background
\end{abstract} subtraction, Feature extraction

\section{Introduction}

In Morocco, the road is the most significant support for people mobility and transport of goods. The road is the first mode of transportation for $90 \%$ of individuals and $75 \%$ of products. Every year, road network continues to grow thanks to the high priority allowed at the national level to road infrastructures. Against this remarkable development of infrastructures, road traffic control and analysis remains modest. Traffic surveillance systems are usually based on the following control tools: Portable radars, patrol radars, and fixed radars (Un-Habitat, 2013). These systems do not allow to intervening immediately on the scene of the misdemeanour, and do not provide the necessary information for operators to ensure the fluidity, the reliability and the safety of displacements, without skipping that these systems are expensive in installation and maintenance (Barendregt and Van Der Kemp, 1988; Mimbela and Klein, 2000). Thus, many problems appear at the level of road traffic circulation such as traffic jams and accidents.

The application of video surveillance techniques in road traffic analysis can assure good results compared to traditional systems (Kastrinaki et al., 2003). Based on surveillance cameras, video surveillance systems can be a valuable tool to manage all tasks related to road traffic. Based on the information which may be collected using these systems, vehicle counting and tracking in real time becomes less complicated and more efficient.

The contribution to solve the problems presented previously was our motive to design a video surveillance system of road traffic. Based on a stationary camera on the road, our real time system can generate a set of information about vehicles flow which crosses the road. The produced results will be useful to take necessary security measures to ensure circulation fluidity. The performance of a real-time system is related to its capacity to detect vehicles and recognize their types as well as its robustness. Consequently, we have established a set of techniques designed to increase vehicle detection and recognition accuracy.

The developed method in this paper starts with a segmentation module of frames coming from the camera. This module is based on background subtraction as a first step, followed by filtering techniques to reduce segmentation noise. In the second module, we extract a set of characteristics related to the shape of moving objects in order to characterize and classify these objects. Vehicle counting and tracking are the goals of the last module.

The rest of paper is organized as follows. The following section presents related work. Section 3 shows a conception of the method which describes the different modules used to count and track vehicles. Section 4 describes the results achieved and their comparison with some existing methods. Finally, section 5 is dedicated to draw a general conclusion.

\section{Literature review}

During the last decade, traffic surveillance systems have become among the leading applications of video surveillance at the planetary level due to vehicle traffic increase. Thus, numerous studies were 
conducted in this field. The primary purpose of most traffic monitoring systems includes the following tasks: Motion detection, vehicle classification, vehicle counting and tracking. The motion detection phase aims to segment the regions of interest based generally on two methods: (1) Background subtraction (Wan et al., 2014; Sobral and Vacavant, 2014); (2) Optical flow (Frias-Velazquez et al., 2015; Chauhan and Krishan, 2013). Classification module aims to classify vehicles to their appropriate type. Most of classification methods are based on feature extraction (Hsieh et al., 2006; Jung and Ho, 1999) from moving objects. The extracted features are usually related to the moving object shape, colour, and texture. Ince (2011) proposes a vehicle counting method based on background subtraction and invariant moments. A mixture of Gaussian models is used to perform the foreground estimation. Vehicle classification is performed by computing moment invariant features of the foreground mask. In (Chen et al., 2007), T.H. Chen proposes a vehicle counting system based on three modules: Object segmentation, blob analysis, and vehicle tracking. This system in (Lai et al., 2010) use a three-step technique comprised of vehicle extraction, vehicle tracking, and classification. The method uses background subtraction to extract the foreground mask containing vehicles region. Filtering and shadow removal are then performed based on geometric parameters. After vehicle detection and tracking, vehicle classification is realized using two shape features called aspect ratio and level of compactness.

Vehicle video surveillance systems know certain constraints due to various problems such as shadows, illumination conditions and occlusions. Numerous studies were proposed to reduce the influence of these problems on the efficiency of vehicle detection and classification systems. In (Tian et al., 2015; Satzoda and Trivedi, 2016; Reilly et al., 2013; Sanin et al., 2012), the authors present a survey and a comparative study of shadow removal methods. The analysed methods are classified to four categories: texture, physical, chromaticity, and geometry. In (Faro et al., 2011), a vehicle detection and tracking system is proposed. The system utilizes luminosity sensors and one fixed camera. The proposed method can handle illumination changes and occlusions based on luminosity information and the features of the detected vehicles. Kanhere and Birchfield (2008) propose a real-time segmentation and tracking system. The system operates at low camera angles using stable features. Based on the feature points, the method can detect and track the partially occluded objects.

\section{Proposed system}

The system consists of three parts: Image segmentation, feature extraction, vehicle counting and tracking. The image segmentation starts with motion detection using background subtraction to eliminate the static background which contains the stationary objects in the road. This step is followed by a set of filtering techniques of segmentation mask to remove the noisy and the blurry image. Canny edge detector is then performed to detect the contours in image sequence. The detected contours will be subject of a box modelling method.

In feature extraction module, we will characterize the boxes associated with vehicles by a set of parameters. This step aims to perform two important tasks:

- Classify vehicles according to their types.

- Detect partial occlusions of vehicles.

Classification process is based on preliminary study applied to a vehicle image database in order to determine feature values associated with vehicle types. Thus, each vehicle detected in the first phase is classified depending on the values of the calculated parameters. In the last module, these vehicles are counted during their movement in the scene.

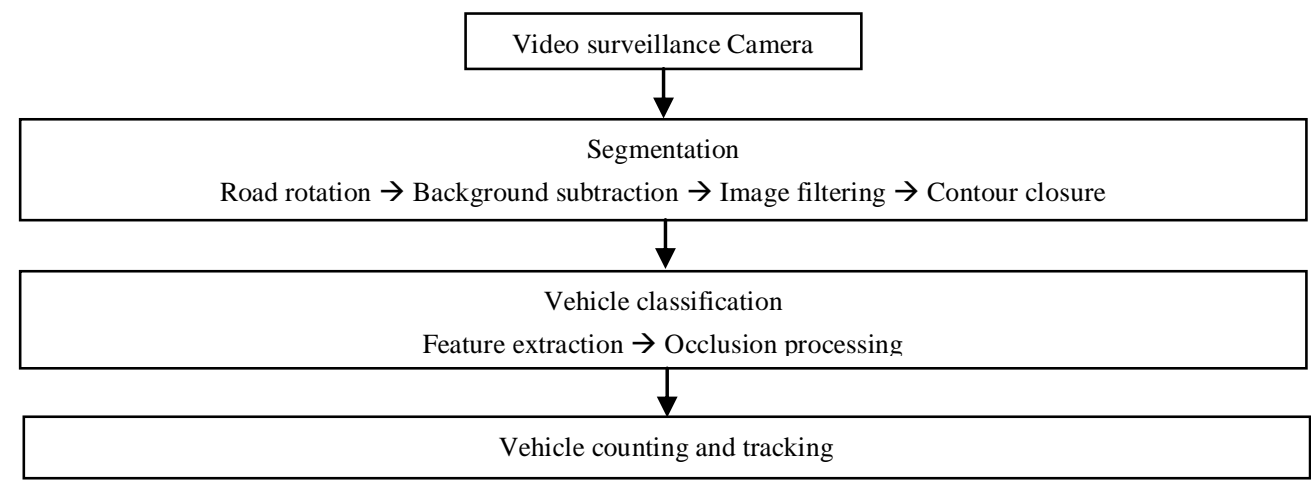

Figure 1. Flowchart of the proposed system 


\subsection{Video sequence rotation}

Road inclination angle compared to vision field axis of surveillance camera influences on the vehicle detection efficiency. Indeed, for some camera positions, it is possible that a false detection can reproduce. A preliminary study is performed to define the inclination angle associated to the highest detection rate. The result of this study showed that when road direction coincides with the axis of vision field of surveillance camera, we have the highest detection rate.

The rotation algorithm is composed of following steps:

- Detection of roadsides direction using Hough transforms (Ballard, 1981). This operation will be applied one time and only in first frame.

- Angle computation between detected roadside and the vision axis of surveillance camera.

- Video sequence rotation respecting that road direction coincides with the axis of vision.

(a)

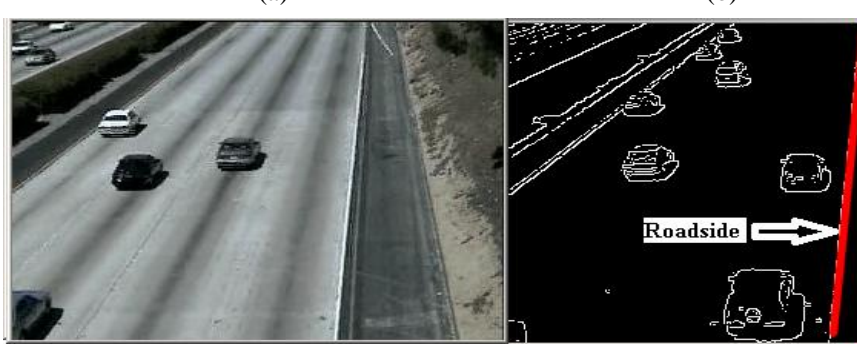

(c)

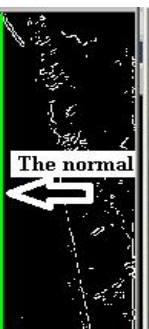

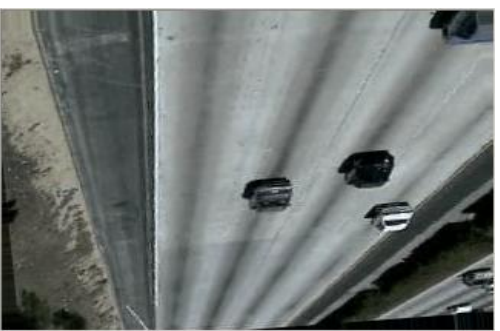

Figure 2. Video rotation: (a) First frame, (b) Line detection using Hough Transform, (c) Rotation result

\subsection{Image segmentation}

\subsubsection{Background subtraction}

In many road traffic video surveillance systems, motion detection process in a video sequence is based mostly on background subtraction. The basic idea of the concept is to determine a model of background corresponding to static regions of video sequence by training. The difference between background model and observed image is then performed to bring out mobile elements in a foreground mask. In our case, the camera is stationary, which means that the background model contains the static objects in the scene (road, trees, and road signs). The foreground mask contains the moving objects.

Numerous subtraction background methods exist in the literature (Wang et al., 2012; Kim et al., 2005). Among these methods, we used Mixture of Gaussian Model (Stauffer and Grimson, 1999). Taking into consideration the multi-modal aspect, Stauffer et al. propose a method based on modelling with a mixture of $\mathrm{N}$ Gaussian for each pixel with $2 \leq \mathrm{N} \leq 5$. For $\mathrm{n}=\{1, \ldots, \mathrm{N}\}$, each element of Gaussian mixture is represented by an average $\mu_{n}$, standard deviation $\sigma_{n}$ and a weight $\alpha_{n}\left(\sum_{n} \alpha_{n}=1\right)$. Foreground mask contains some amount of noise due to segmentation step. To remove the noisy and blurry image, morphological operations, such as opening and closing, are applied.
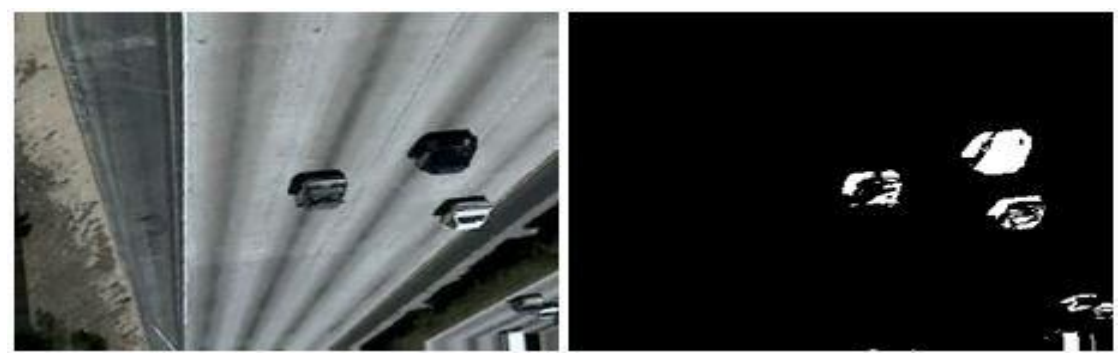

Figure 3. Background subtraction using Mixture of Gaussian method

\subsubsection{Filtering and contour detection}

Segmentation mask still contains residual noise. That can have an adverse impact on vehicle detection and tracking rate. To eliminate this noise, we propose to model the detected contours with boxes. For that purpose, we use firstly Canny edge detector (Canny, 1986) to detect vehicle contours. Then, we compute the coordinates of contours. The coordinates is then used to draw boxes. We obtain a mask containing vehicles modelled by boxes (Fig. 4). 
(a)

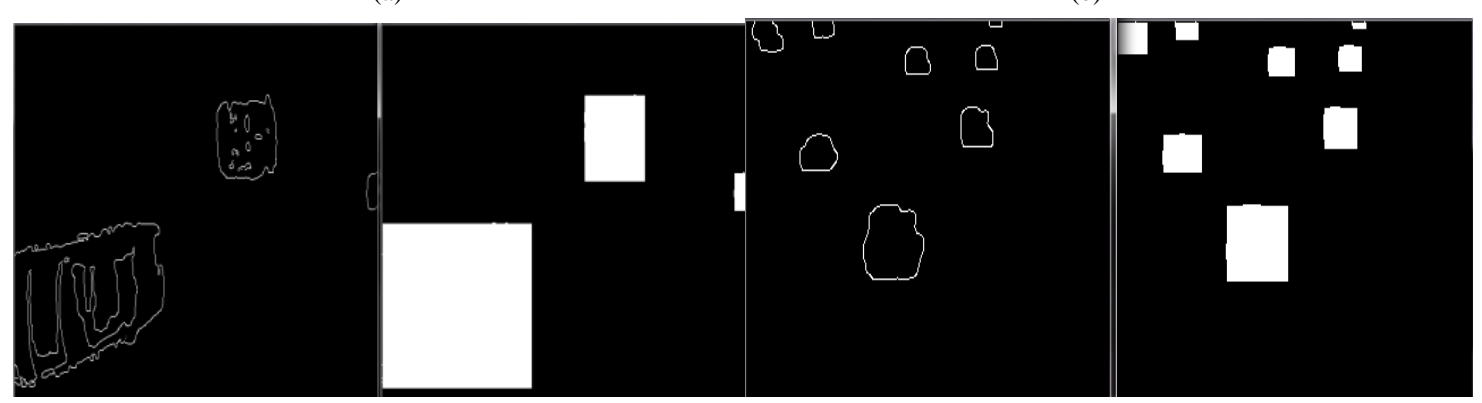

Figure 4. Results of box modelling technique

(a): Single-directional situation 1; (b): Single-directional situation 2

\subsection{Vehicle classification}

In classification process, the first task consists of classifying vehicles to their types based on extracted features from vehicle shape. An approach to detect and eliminate the partial occlusions is also performed. This approach is added to classification process in order to improve vehicle counting efficiency.

Feature extraction of vehicles can be performed using several parameters such as the shape (Ali et al., 2013; Huang et al., 2012), the colour, and the texture. In our system, we focused on shape to establish two parameters which can describe the moving vehicles. The two parameters used are related to length and width of detected boxes in segmentation phase. That means that the values of parameters vary according to detected vehicle type.

$\mathrm{R} 1=\frac{\text { Height }}{\text { Width }}$,

$\mathrm{R} 2=\frac{((\text { Height }+ \text { Width }) * 2)^{2}}{(\text { Height } * \text { Width })}$.

In the above equations, Height, Width denote the height, width, of the detected box, respectively. The parameters R1 and R2 present two different combinations of Height and Width in order to establish parameters characterizing each moving object. The choice of combinations is related to the stability against vehicle movement in the scene and the variation according to the vehicle type.

Based on preliminary study applied to a vehicle image database containing 6500 images as shown in Figure 5, we were able to analyze the variations of parameters R1 and R2 depending on various classes of vehicles. We conclude that the values of parameters vary on defined intervals according to vehicle type (Bike, car), except when there is a vehicle occlusion (case 1 and case 2 in Table 1).

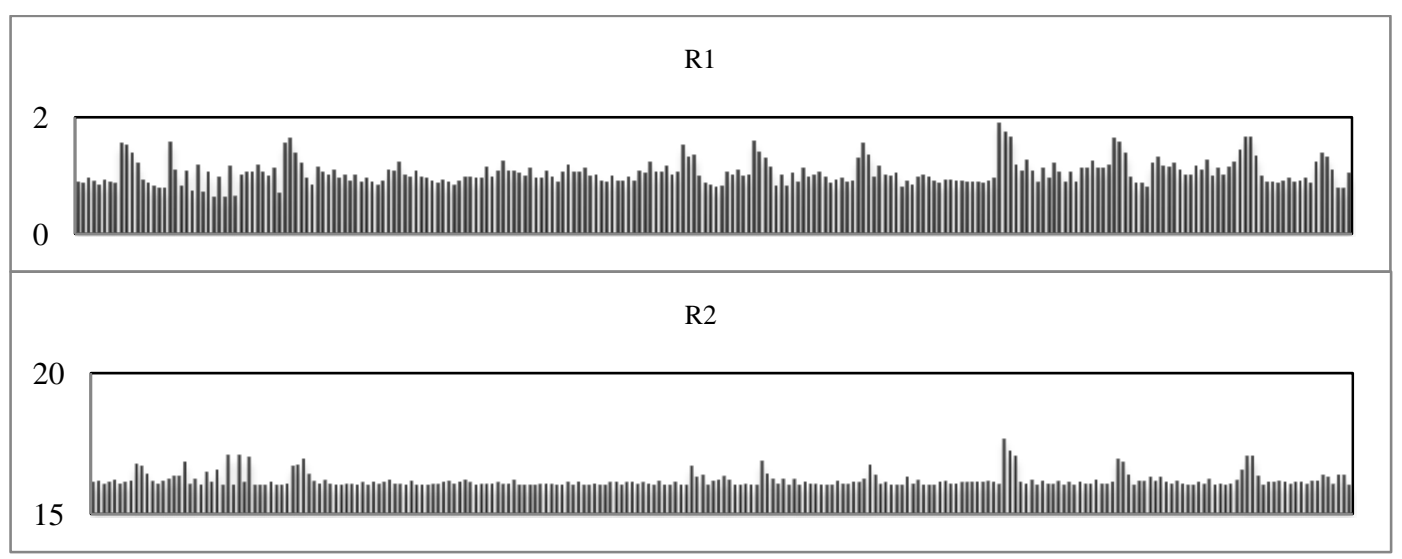

Figure 5. Results of the preliminary study 
Table 1. Classification intervals

\begin{tabular}{|l|l|l|l|}
\hline Vehicle type & Sample Image & R1 & R2 \\
\hline Car & & $0.8-1.3$ & $16-16.5$ \\
\hline Bike & & $1.5-1.8$ & $17-18.5$ \\
\hline Case 1 & & & 17.04 \\
\hline Case 2 & & 0.603 & \\
\hline & & & \\
\hline
\end{tabular}

We concluded that when R2 value belongs to the interval (16-16.5), the moving vehicle is a car. When the value of R2 belongs to the interval (17-18.5), the moving vehicle is a bike. Otherwise, if R2 value does not belong to any determined interval previously, there is one or more vehicle occlusion present in the scene. In this situation, we analyse R1 value. If R1 value is higher than 1.3, the box associated with the partial occlusion will be divided into two boxes based on height. Otherwise, we divide the box based on width (Fig. 6).

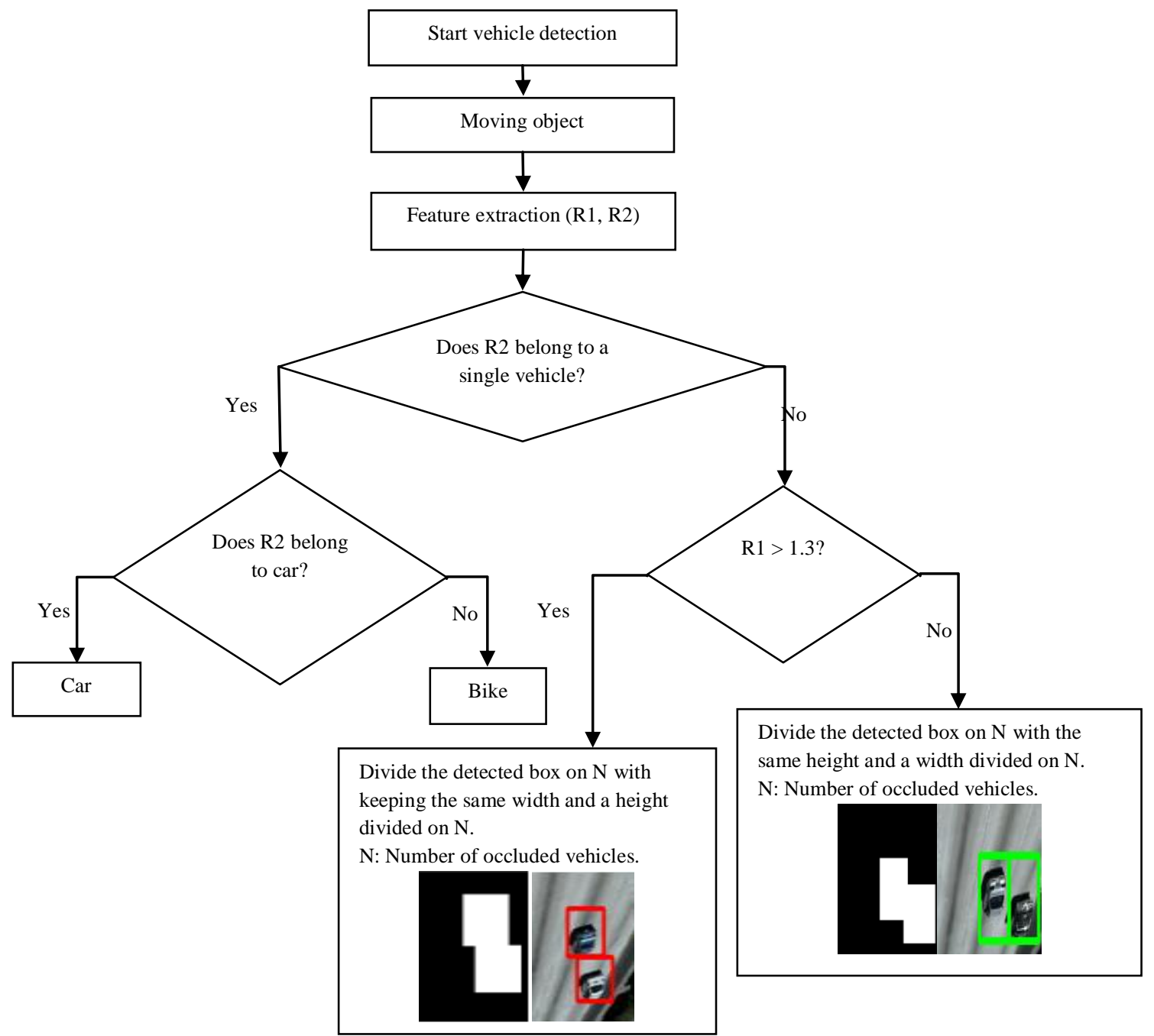

Figure 6. Flowchart of vehicle classification module 


\subsection{Vehicle counting and tracking}

Figure 7 reveals the established zone for vehicle video surveillance. Two lines A and B present the borders of the zone. Every vehicle passing through this area will be counted and tracked.

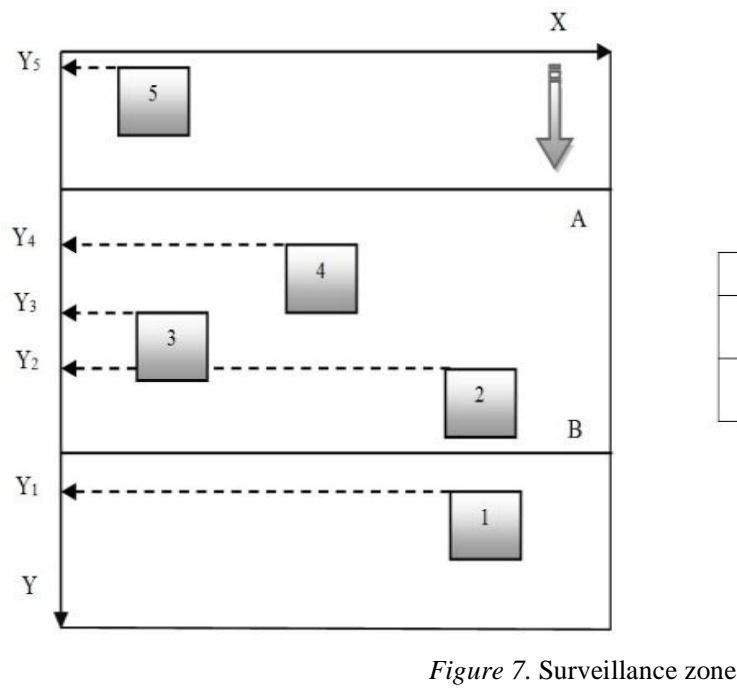

The process consists of knowing at any moment the number of vehicles crossing surveillance zone and their positions according to axis (OY). Segmentation module calculates these positions and save them in a one dimensional table. Thus, we can conclude recent and previous positions of each vehicle and if a new vehicle is present on the road. Vehicle counting process will depend on this result. Based on classification phase, we introduce two variables. Each variable is used to count the number of a defined type of vehicles.

In the tracking and counting system, the table comparison is the most important module. The inputs of this block are the table (Fig. 8) and V the current number of objects in the surveillance area AB, $\mathrm{V}$ also presents the number of positions added to the table at each frame.

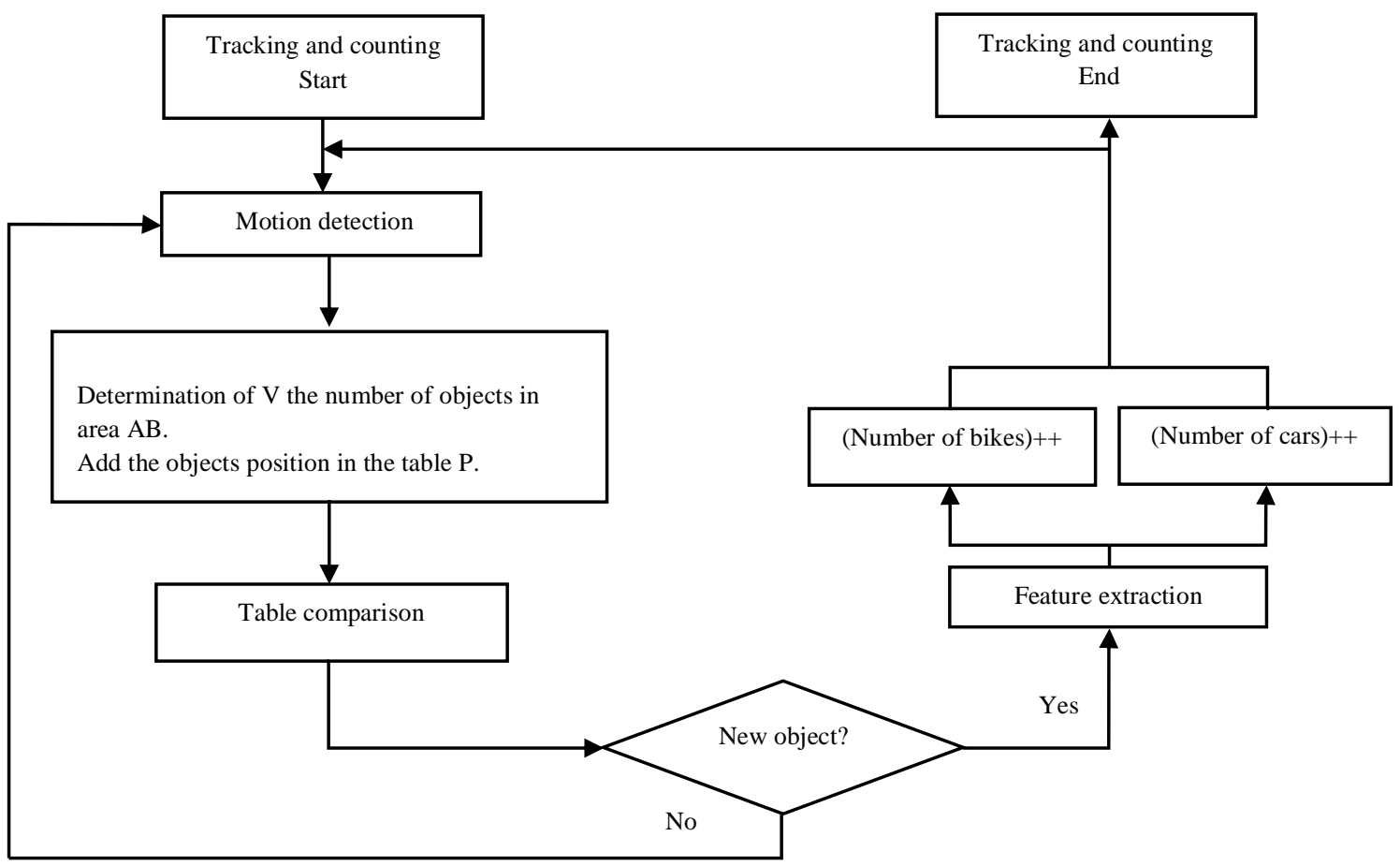

Figure 8. Illustration of proposed method in counting and tracking module 


\section{Experimental results}

\subsection{System implantation}

Proposed System was tested in different video sequences. The difference between tested videos appears in several parameters such as distance between road and camera, road inclination angle and illumination conditions. Figure 9 presents the results obtained during realized system operation. Blue segments define the surveillance zone. The provided bounding boxes take two colours depending on the type of detected vehicles. Above these boxes appears a number indicating the number of vehicles passing through the road since the start of the algorithm execution.

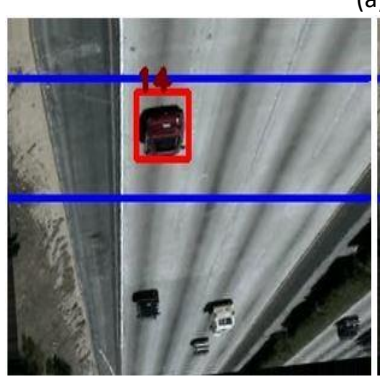

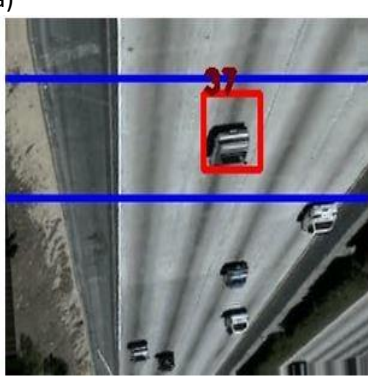

(b)

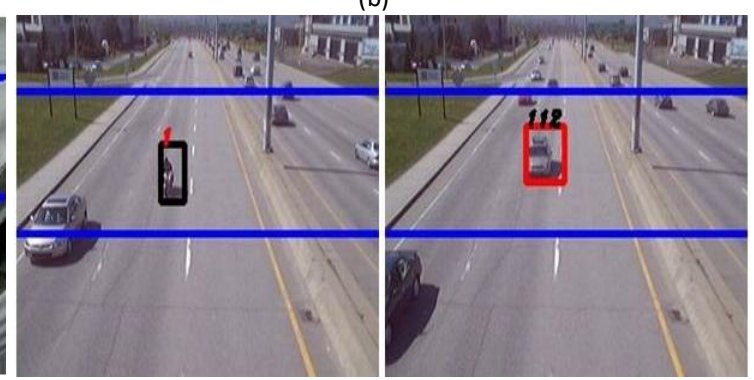

Figure 9. Results of proposed method
(a) Video 1
(b) Video 2

\subsection{System test in GRAM-RTM dataset}

GRAM-RTM dataset (Guerrero-Gomez-Olmedo et al. 2013) is a benchmark for multi-vehicles tracking in real-time. Certain changes were made to the system to adapt it to GRAM-RTM dataset. Such as the video surveillance zone this will be defined using a mask (Fig. 10).

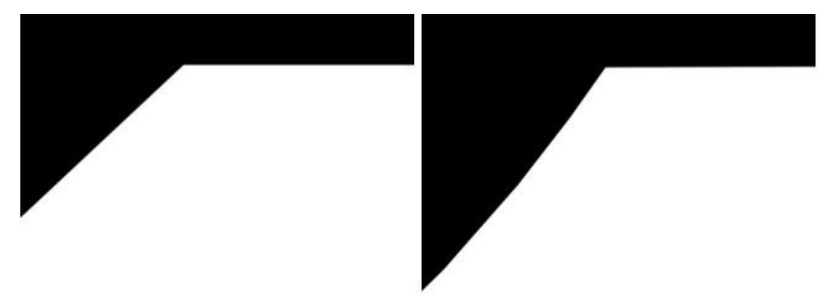

Figure 10. Mask used to define the surveillance zone

The intervals of the coefficients R1 and R2 associated with the different classes determined will vary. The implementation of the system in another scene with a different position of camera involves this change because the both coefficients have a direct relation to the height and width of the detected vehicles. Thus, the classification intervals defined to classify the cars, bikes and partial occlusions will take new values as is shown in (Table 2).

Table 2. Classification intervals for GRAM dataset

\begin{tabular}{|c|c|c|c|c|}
\hline Video test & Classes & Image Sample & R1 & R2 \\
\hline \multirow[t]{2}{*}{ Video 1} & Car & $1=$ & $0.2-0.6$ & $15-16$ \\
\hline & Bike & & $1.6-2.5$ & $17-19$ \\
\hline \multirow[t]{2}{*}{ Video 2} & Car & & $0.5-1.4$ & $15-17.5$ \\
\hline & Occlusion & & 1.75 & $17.5-25$ \\
\hline
\end{tabular}


Figure 11 presents the results obtained during the execution of the realized system. The provided bounding boxes take two colours relatively to the type of the detected vehicle. Above these rectangles appears a number indicating the number of vehicles passing through the scene.

(a)

(b)

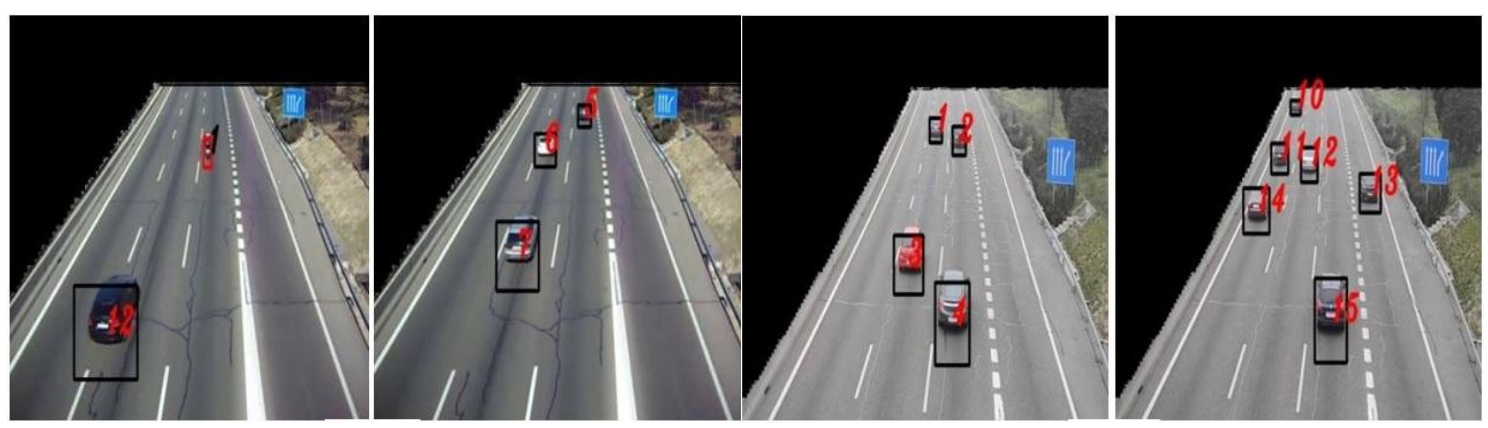

Figure 11. Results of proposed method in GRAM dataset

(a) Video 1; (b) Video 2

\subsection{Performance evaluation}

\subsubsection{Vehicle counting accuracy}

An analytical study was conducted to calculate the vehicle counting accuracy to test the efficiency of our system. Table 3 presents the results calculated at each block of the segmentation phase and after the technique of partial occlusion elimination based on feature extraction. The average accuracy measured in the test conducted on three different roads had as value $98.7 \%$.

Table 3. Vehicle counting accuracy in different scenes

\begin{tabular}{|l|l|l|l|l|l|}
\hline Video test & $\begin{array}{l}\text { Real number } \\
\text { of vehicles }\end{array}$ & $\begin{array}{l}\text { Vehicles number } \\
\text { using Canny }\end{array}$ & $\begin{array}{l}\text { Vehicles number } \\
\text { with box modelling }\end{array}$ & $\begin{array}{l}\text { Vehicles number } \\
\text { after classification }\end{array}$ & Accuracy \\
\hline $\begin{array}{l}1^{\text {st }} \text { video } \\
\text { sequence }\end{array}$ & 5 & 30 & 6 & 5 & $100 \%$ \\
\hline $\begin{array}{l}2^{\text {nd }} \text { video } \\
\text { sequence }\end{array}$ & 38 & 45 & 36 & 37 & $97.36 \%$ \\
\hline $\begin{array}{l}3^{\text {rd }} \text { video } \\
\text { sequence }\end{array}$ & 162 & 176 & 170 & 160 & $98.76 \%$ \\
\hline Average & \multicolumn{3}{|l|}{} & $98.7 \%$ \\
\hline
\end{tabular}

\subsubsection{Comparative methods}

The robustness of any method is not related only to the precision of the realized system but also to the comparison of the results with other existing methods which treat the same field. Table 4 presents the result of this comparison.

Table 4. Results of comparative study

\begin{tabular}{|l|l|l|l|l|l|}
\hline Comparative methods & $\begin{array}{l}\text { (Huang } \text { et al., } \\
\text { 2012) }\end{array}$ & (Lei et al., 2008) & $\begin{array}{l}\text { (Pornpanomchai } \\
\text { et al., 2008) }\end{array}$ & $\begin{array}{l}\text { (Mohana } \\
\text { et al., 2009) }\end{array}$ & $\begin{array}{l}\text { Proposed } \\
\text { method }\end{array}$ \\
\hline Studied types of vehicles & Cars and bikes & Cars & Cars & $\begin{array}{l}\text { Cars } \\
\text { bikes }\end{array}$ \\
\hline Segmentation methods & $\begin{array}{l}\text { Background } \\
\text { subtraction }\end{array}$ & $\begin{array}{l}\text { Background } \\
\text { subtraction }\end{array}$ & $\begin{array}{l}\text { Background } \\
\text { subtraction }\end{array}$ & Optical flow & $\begin{array}{l}\text { Background } \\
\text { subtraction }\end{array}$ \\
\hline Classification methods & $\begin{array}{l}\text { Feature } \\
\text { extraction }\end{array}$ & X & X & $\begin{array}{l}\text { Feature } \\
\text { extraction }\end{array}$ \\
\hline $\begin{array}{l}\text { Measured accuracy of } \\
\text { vehicle counting }\end{array}$ & $98.6 \%$ & $\begin{array}{l}\text { Scene 1: } 90.41 \% \\
\text { Scene 2: } 70.31 \% \\
\text { Scene 3: } 98.39 \% \\
\text { Scene 4: } 80.00 \%\end{array}$ & $\begin{array}{l}\text { Afternoon: } 91.98 \% \\
\text { Evening: } 96.35 \%\end{array}$ & $94.04 \%$ & $98.7 \%$ \\
\end{tabular}




\section{Conclusions}

This paper has proposed a real-time vehicle analysis and counting system for vehicle video surveillance. The proposed system is able to detect, locate, and automatically recognize vehicles in video sequences. In other words, the system can measure the quantity and characteristics of vehicles in real-time based on three modules, segmentation, detection and classification.

Despite the considerable success in the segmentation process, there are some problems during the experiments that cannot be overlooked for performance evaluation. Contrary to the preliminary hypothesis, the effects of inhomogeneous vehicle architecture seem to play an important role in noise generation during the detection phase. This led us back to apply a mask for closing contours to eliminate such disturbances. Thus, the quality of filtering is increased considerably which leads to an optimization in the overall efficiency of the system.

In the classification phase, the system is able to classify vehicles into two classes (car, bike) using feature extraction. However, some difficulties are observed during the experiments because of vehicle occlusions. The extracted features are used also to classify vehicle occlusions in order to eliminate them. As a result, the designed system has successfully eliminated the majority of vehicle occlusions.

The results of the proposed method reached a value of $98.7 \%$ as average measured at three different scenes. Which indicates that the system is efficient as a reliable vehicle counting tool for traffic video surveillance systems. In addition, the system is characterized by reasonable robustness against illumination changes and objects size. Nevertheless, the provided results demonstrate that the counting methodology in traditional road traffic video surveillance systems based on the camera can be improved in terms of accuracy by implementing the proposed method of detection and classification.

Further studies could be conducted in the future to demonstrate the effectiveness of the proposed method on a much larger test scale. Following this work, many perspectives are possible, the most important being without doubt the realization of an embedded system based on FPGA in order to test the entire system in road scenes in real time and for long periods.

\section{References}

1. Ali, M.H., Kurokawa, S., and Shafie, A. (2013) Autonomous road surveillance system: A proposed model for vehicle detection and traffic signal control. Procedia Computer Science, 19, 963-970.

2. Ballard, D.H. (1981) Generalizing the Hough transform to detect arbitrary shapes. Pattern Recognition, 13, 111-122.

3. Barendregt, A.J., and Van Der Kemp, M.J. (1988). U.S. Patent No. 4,786,913. Washington, DC: U.S. Patent and Trademark Office.

4. Canny, J. (1986) A computational approach to edge detection. IEEE Transactions on pattern analysis and machine intelligence, pp. 679-698.

5. Chauhan, A.K., and Krishan, P. (2013) Moving object tracking using Gaussian mixture model and optical flow. International Journal of Advanced Research in Computer Science and Software Engineering, 3(4).

6. Chen, T.-H., Lin, Y.-F., and Chen, T.-Y., (2007) Intelligent vehicle counting method based on blob analysis in traffic surveillance. In Innovative Computing, Information and Control, 2007. ICICIC'07. Second International Conference, pp. 238-238).

7. Faro, A., Giordano, D., and Spampinato, C. (2011) Adaptive background modelling integrated with luminosity sensors and occlusion processing for reliable vehicle detection. IEEE Transactions on Intelligent Transportation Systems, 12, 1398-1412.

8. Frias-Velazquez, A., Van Hese, P., Pizurica, A., and Philips, W. (2015) Split-and-match: A bayesian framework for vehicle reidentication in road tunnels. Engineering Applications of Artificial Intelligence, 45, 220-233.

9. Guerrero-Gomez-Olmedo, R., Lopez-Sastre, R.J., Maldonado-Bascon, S., and Fernandez-Caballero, A. (2013) Vehicle tracking by simultaneous detection and viewpoint estimation. In IWINAC 2013, Part II, LNCS 7931, pp. 306-316.

10. Hsieh, J.-W., Yu, S.-H., Chen, Y.-S., and Hu, W.-F. (2006) Automatic traffic surveillance system for vehicle tracking and classification. IEEE Transactions on Intelligent Transportation Systems, 7, 175187.

11. Huang, D.-Y., Chen, C.-H., Hu, W.-C., Yi, S.-C., Lin, Y.-F. (2012) Feature-based vehicle flow analysis and measurement for a real-time traffic surveillance system. Journal of Information Hiding and Multimedia Signal Processing, 3, 279-294. 
12. Ince, E., (2011) Measuring traffic flow and classifying vehicle types: A surveillance video based approach. Turkish Journal of Electrical Engineering and Computer Sciences, 19, 607-620.

13. Jung, Y.-K., and Ho, Y.-S. (1999) Traffic parameter extraction using video-based vehicle tracking. In Intelligent Transportation Systems, 1999. Proceedings. 1999 IEEE/IEEJ/JSAI International Conference, pp. 764-769.

14. Kanhere, N.K., and Birchfield, S.T. (2008) Real-time incremental segmentation and tracking of vehicles at low camera angles using stable features. IEEE Transactions on Intelligent Transportation Systems, 9, 148-160.

15. Kastrinaki, V., Zervakis, M., and Kalaitzakis, K. (2003) A survey of video processing techniques for traffic applications. Image and vision computing, 21(4), pp. 359-381.

16. Kim, K., Chalidabhongse, T.H., Harwood, D., and Davis, L. (2005) Real-time foreground-background segmentation using codebook model. Real-time imaging, 11, 172-185.

17. Lai, J.-C., Huang, S.-S., and Tseng, C.-C., (2010) Image-based vehicle tracking and classification on the highway. In Green Circuits and Systems (ICGCS), 2010 International Conference, pp. 666-670.

18. Lei, M., Leoch, D., Gouton, P., and Madani, K. (2008) A video-based real-time vehicle counting system using adaptive background method. In Signal Image Technology and Internet Based Systems, 2008. SITIS'08. IEEE International Conference, pp. 523-528).

19. Mimbela, L.E.Y., and Klein, L.A. (2000) Summary of vehicle detection and surveillance technologies used in intelligent transportation systems. Las Cruces, NM: The Vehicle Detector Clearinghouse, New Mexico State University.

20. Mohana, H., Ashwathakumar, M., and Shivakumar, G. (2009) Vehicle detection and counting by using real time traffic ux through differential technique and performance evaluation. In Advanced Computer Control, 2009. ICACC'09. International Conference on IEEE, pp. 791-795.

21. Moutakki, Z., Ouloul, I., Afdel, K., Amghar, A. (2017) Real-Time Video Surveillance System for Traffic Management with Background Subtraction Using Codebook Model and Occlusion Handling. Transport and Telecommunication Journal, 18(4), pp. 297-306. Retrieved 24 Dec. 2017, from doi:10.1515/ttj-2017-0027.

22. Pornpanomchai, C., Liamsanguan, T., and Vannakosit, V. (2008) Vehicle detection and counting from a video frame. In Wavelet Analysis and Pattern Recognition, 2008. ICWAPR'08. International Conference on IEEE, 1, pp. 356-361.

23. Reilly, V., Solmaz, B., and Shah, M. (2013) Shadow casting out of plane (scoop) candidates for human and vehicle detection in aerial imagery. International Journal of Computer Vision, 101, 350366.

24. Sanin, A., Sanderson, C., and Lovell, B.C. (2012) Shadow detection: A survey and comparative evaluation of recent methods. Pattern Recognition, 45, 1684-1695.

25. Satzoda, R.K., and Trivedi, M.M. (2016) Multipart vehicle detection using symmetry-derived analysis and active learning. IEEE Transactions on Intelligent Transportation Systems, 17, 926-937.

26. Sobral, A., and Vacavant, A. (2014) A comprehensive review of background subtraction algorithms evaluated with synthetic and real videos. Computer Vision and Image Understanding, 122, pp. 4-21.

27. Stauffer, C., and Grimson, W.E.L. (1999) Adaptive background mixture models for real-time tracking. In Computer Vision and Pattern Recognition, 1999. IEEE Computer Society Conference, pp. 246-252.

28. Tian, B., Tang, M., and Wang, F.-Y. (2015) Vehicle detection grammars with partial occlusion handling for traffic surveillance. Transportation Research Part C: Emerging Technologies, 56, 80-93.

29. Un-Habitat. (2013) Planning and design for sustainable urban mobility: Global report on human settlements 2013. Routledge.

30. Wan, Y., Huang, Y., and Buckles, B. (2014) Camera calibration and vehicle tracking: Highway traffic video analytics. Transportation Research Part C: Emerging Technologies, 44, 202-213.

31. Wang, Y., Liang, Y., Zhang, L., and Pan, Q. (2012) Adaptive spatiotemporal background modelling. IET Computer Vision, 6, 451-458. 